\title{
More on the flavor dependence of $m_{\varrho} / f_{\pi}$
}

\author{
Andrey Yu. Kotov, ${ }^{a}$ Daniel Nogradi, ${ }^{b}$ Kalman K. Szabo ${ }^{a, c}$ and Lorinc Szikszai ${ }^{b}$ \\ ${ }^{a}$ Julich Supercomputing Centre, Forschungszentrum Julich, \\ Wilhelm-Johnen-Strasse, Julich D-52425, Germany \\ ${ }^{b}$ Eötvös University, Institute for Theoretical Physics, \\ Pazmany Peter setany 1/a, Budapest 1117, Hungary \\ ${ }^{c}$ Department of Physics, Wuppertal University, \\ Gaussstrasse 20, 42119, Germany \\ E-mail: a.kotov@fz-juelich.de, nogradi@bodri.elte.hu, \\ k.szabo@fz-juelich.de, szikszail@caesar.elte.hu
}

ABSTRACT: In previous work, [arXiv:1905.01909], we have calculated the $m_{\varrho} / f_{\pi}$ ratio in the chiral and continuum limit for $\mathrm{SU}(3)$ gauge theory coupled to $N_{f}=2,3,4,5,6$ fermions in the fundamental representation. The main result was that this ratio displays no statistically significant $N_{f}$-dependence. In the present work we continue the study of the $N_{f}$-dependence by extending the simulations to $N_{f}=7,8,9,10$. Along the way we also study in detail the $N_{f}$-dependence of finite volume effects on low energy observables and a particular translational symmetry breaking unphysical, lattice artefact phase specific to staggered fermions.

KEYwords: Lattice QCD, Lattice Quantum Field Theory

ARXiv EPrint: 2107.05996 


\section{Contents}

1 Introduction and summary 1

2 Discretization and unphysical phases with staggered fermions 2

3 Finite volume effects 4

$\begin{array}{lll}4 & \text { Chiral-continuum extrapolation } & 7\end{array}$

$\begin{array}{llr}5 & \text { Conclusion } & 9\end{array}$

\section{Introduction and summary}

We study the flavor number dependence of the ratio of the vector meson mass and the pseudoscalar decay constant in $\mathrm{SU}(3)$ gauge theory. The ratio is significant for a large class of beyond Standard Model theories envisioning a strongly interacting Higgs sector and a composite Higgs boson [1]. The elementary fermion ingredients of the composite Higgs boson may form other bound states, such as a vector meson, which would be one of the new, so far undetected, particles the theory predicts. The pseudoscalar decay constant sets the scale, in many theories it is simply identified with $v=246.22 \mathrm{GeV}$, the symmetry breaking scale of the Standard Model. Having non-perturbative results for $m_{\varrho} / f_{\pi}$ then determines the vector meson mass $m_{\varrho}$ in physical units. This beyond Standard Model scenario, and variants thereof, attracted enormous interest in the lattice community in the past decade [2-34]. For a recent reviews of the available lattice results see [35] and references therein.

Apart from the phenomenological motivation the $N_{f}$-dependence of our ratio is an interesting QFT question on its own. Once both $f_{\pi}$ and $m_{\varrho}$ are understood to be defined at finite fermion mass $m$ and the chiral limit is only taken for the ratio, $m_{\varrho} / f_{\pi}$ is a meaningful quantity both inside and outside the conformal window. Outside the conformal window both the denominator and nominator are finite in the chiral limit with an obviously finite ratio. Inside the conformal window both $m_{\varrho}$ and $f_{\pi}$ behave as $O\left(m^{\alpha}\right)$ for small $m$ with the same exponent $\alpha$, again leading to a finite ratio in the chiral limit. Hence the ratio is meaninful and well-defined on the full range $0 \leq N_{f} \leq 16$, including the quenched case $N_{f}=0$ and the last integer flavor number $N_{f}=16$ before asymptotic freedom is lost at $N_{f}=33 / 2$. Formally, $N_{f}=33 / 2$ corresponds to a free theory [36] and as such $m_{\varrho}=2 m$ and $f_{\pi}=\sqrt{12} m$, leading to $m_{\varrho} / f_{\pi}=1 / \sqrt{3}$. This is an order of magnitude smaller than $\sim 8$ found for $2 \leq N_{f} \leq 6$. Hence on the range $7 \leq N_{f} \leq 16$ the ratio will drop an order of magnitude and it is not a priori known whether the drop will be gradual or rapid, nor is it known if the onset of the conformal window somewhere around $10 \leq N_{f} \leq 13$ is connected to it in any way. 
Motivated by both the phenomenological implications and the purely QFT aspects we continue the investigation with $7 \leq N_{f} \leq 10$ in the present work. Even though we would like to know the behavior for $11 \leq N_{f} \leq 16$ as well, finite volume effects are growing as a function of $N_{f}$ so rapidly that unfortunately we must postpone these flavor numbers to future work.

The organization of the paper is as follows. In section 2 we first study the $N_{f^{-}}$ dependence of an unphysical lattice phase specific to staggered fermions. The reason for doing so is that as $N_{f}$ grows the size of the unphysical phase in the $(\beta, m)$ plane grows and one must avoid it in order to perform the physically relevant chiral-continuum limit. Section 3 details our study of the finite volume effects, the upshot of which is that as $N_{f}$ is growing so do finite volume effects. In fact the growth is rather rapid and is the main reason $N_{f}=10$ is the highest flavor number we can reliably simulate at the moment. The chiral-continuum limit is investigated in section 4 once the bare parameters are chosen such that unphysical phases are avoided and finite volume effects are suppressed sufficiently. We end with conclusions and possible outlook to future work in section 5.

\section{Discretization and unphysical phases with staggered fermions}

The lattice discretization in the present work follows exactly [37]; 4 steps of stout smearing $[38,39]$ is applied to naive staggered fermions with smearing parameter $\varrho=0.12$. A combination of the HMC and RHMC algorithms [40, 41] with or without rooting are used to have the desired continuum flavor number $N_{f}$.

Both in [37] and the present work simulations are run at particular points of the $(\beta, m)$ phase diagram at given $N_{f}$. It is important that the bare parameters are all in the region of phase space which is continuously connected to the physical $\beta \rightarrow \infty$ region, especially because unphysical phases do exist with staggered fermions.

The possibility that in the $(\beta, m)$ bare parameter space an unphysical Aoki-like phase might exist with staggered fermions was first pointed out in $[42,43]$. Using staggered chiral perturbation theory it was shown that decreasing the mass on coarse lattices can lead to condensation of taste split meson states which in turn means that the vacuum becomes unstable. The new vacuum has different symmetries from the one expected in the continuum and in particular the so-called staggered shift symmetry, which is a translation by a single site accompanied by a phase factor for fermion fields, is broken. Briefly, taste split meson masses $M^{2}$ in staggered chiral perturbation theory receive a continuum-like contribution from the fermion mass, $O(m)$, but also a contribution from taste splitting operators, $O\left(a^{2}\right)$. If the latter is negative and large in absolute value compared to the former, $M^{2}$ may turn negative, leading to the aforementioned instability. Convincing numerical evidence for this scenario was provided in [44] for $N_{f}=8,12$ and the relationship between the staggered perturbation theory picture and the actual numerical results were further clarified in [45].

In this section we study the unphysical, shift symmetry broken phase with our particular discretization and the full range of flavor numbers $2 \leq N_{f} \leq 10$ contained in both [37] and the present work. The main conclusion will be that even though unphysical phases do 
exist for $N_{f}>2$ and we do map them out, our simulation points are all in the physical phase, justifying our chiral-continuum extrapolations.

The single site shift symmetry in question is [46]

$$
\chi(x) \rightarrow \xi_{\mu}(x) \chi(x+\hat{\mu}), \quad \bar{\chi}(x) \rightarrow \bar{\chi}(x+\hat{\mu}) \xi_{\mu}(x), \quad U_{\mu}(x) \rightarrow U_{\mu}(x+\hat{\mu}),
$$

where $\chi(x)$ is the staggered field at integer site $x, \hat{\mu}$ is the unit vector on the lattice in direction $\mu$ and $\xi_{\mu}(x)=(-1)^{\sum_{\nu>\mu} x_{\nu}}$. In this convention the staggered signs in the Dirac operator are $\eta_{\mu}(x)=(-1)^{\sum_{\nu<\mu} x_{\nu}}$. The staggered action is clearly invariant under this set of transformations.

As discussed in [44] a suitable order parameter for the study of the potential spontaneous breaking of (2.1) is the difference of plaquettes on neighboring sites. More precisely, in terms of the plaquette $P(x)$,

$$
\Delta_{\mu} P=\sum_{x_{\mu} \text { even }}\langle P(x+\hat{\mu})-P(x)\rangle,
$$

where the sum over the lattice involves only even $x_{\mu}$ coordinates. Clearly, if the sum would be over the entire lattice $\Delta_{\mu} P$ would always be zero. In this way $\Delta_{\mu} P$ measures if translational invariance in direction $\mu$ holds for the plaquette or not. In the physical phase, where translational invariance for gluonic observables is present, $\Delta_{\mu} P=0$ for all $\mu$. The unphysical phase will be signaled by $\Delta_{\mu} P \neq 0$ for at least one direction $\mu$.

It is a straightforward exercise to map the observable $\Delta_{\mu} P$ as a function of $(\beta, m)$ for the various flavor numbers. A useful quantity to monitor is the square $\Delta_{\mu} P \Delta_{\mu} P$ involving a sum over $\mu$. Two typical results are shown for $\Delta_{\mu} P \Delta_{\mu} P$ at fixed $\beta$ as a function of $m$ and at fixed $m$ as a function of $\beta$ in figure 1 with $N_{f}=7$ on $18^{4}$ lattices. It is not our goal to obtain very precise values for $\left(\beta_{c}, m_{c}\right)$ corresponding to the spontaneous breaking of translational invariance, for our purposes an estimate will suffice which can be read off from results of the type shown in figure 1. A detailed finite size scaling study would be required for anything more precise. As we will see our simulation points are so far away from the $\left(\beta_{c}, m_{c}\right)$ phase boundaries that a rough estimate is indeed sufficient.

Performing the scans on $12^{4}$ and $18^{4}$ lattices shows that volume dependence is negligible on our level of precision. The summary of our results for the phase boundaries are shown in figure 2 for all flavor numbers where the thickness of the boundaries include the uncertainty related to our crude reading off of $\left(\beta_{c}, m_{c}\right)$ on fixed $18^{4}$ lattice volumes.

For each $N_{f}>2$ a triangle shaped region corresponds to the spontaneously broken shift symmetry phase at finite $(\beta, m)$. This triangle presumably extends down to $m=0$ at two particular $\beta$ values. The bare mass, above which translational symmetry is unbroken for all $\beta$ is a growing function of $N_{f}$ as can be seen in figure 2. Not surprisingly, the particular $\beta$ above which translational symmetry is unbroken for all masses is a decreasing function of $N_{f}$. At $N_{f}=3$ we could not resolve the triangle shape because the broken phase only occures for very small masses, but nevertheless could find a transition. Interestingly, we could not detect any translational symmetry broken phase for $N_{f}=2$, perhaps because no such phase exists or perhaps because it occurs at extremely small masses. 

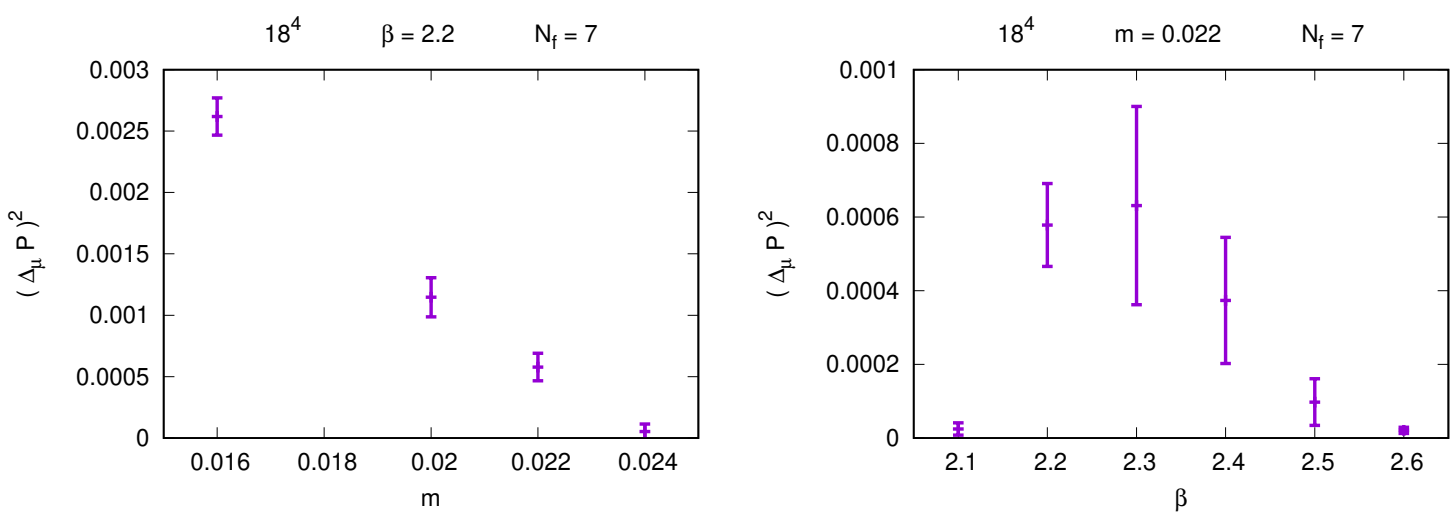

Figure 1. Two examples at $N_{f}=7$ for finding the boundary of the shift symmetry broken phase. The square of the observable (2.2) is shown at fixed $\beta$ (left) and fixed $m$ (right).

Shift symmetry breaking phase boundaries for various $\mathrm{N}_{\mathrm{f}}$

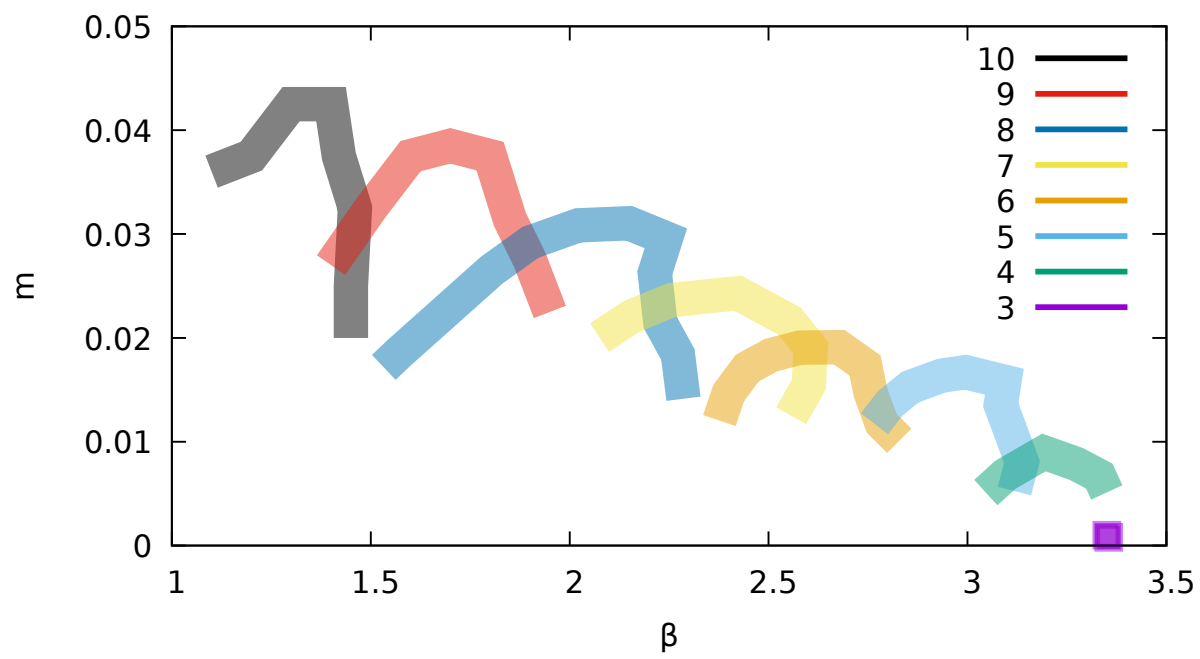

Figure 2. The shift symmetry breaking phase boundaries in the $(\beta, m)$ plane. The broken phase is located under the curves, i.e. for small quark masses. The curves are given with different colors for different number of flavors.

The $(\beta, m)$ values for $N_{f}=2,3,4,5,6$ which were used in the chiral-continuum extrapolations in [37] were listed in tables 3 and 4 of said work while the same parameters are listed in table 3 for the present work with $N_{f}=7,8,9,10$. Clearly, all parameters used for the chiral-continuum extrapolations are in the physical phase and far from the $\left(\beta_{c}, m_{c}\right)$ phase boundaries.

\section{Finite volume effects}

Just as in [37], a prerequisite step before chiral-continuum extrapolations are performed is the study of finite volume effects. The volume, measured in $m_{\pi}$ units, needs to be large enough in order to suppress finite volume distortions of the ratio $m_{\varrho} / f_{\pi}$ especially because as the volume is increasing $m_{\varrho}$ and $f_{\pi}$ are moving in the opposite direction. The finite 


\begin{tabular}{|c|c|c|c|c|c|}
\hline$N_{f}$ & $\beta$ & $m$ & $L / a$ & $a m_{\pi}$ & $a f_{\pi}$ \\
\hline \multirow[t]{5}{*}{7} & 3.00 & 0.0100 & 20 & $0.210(3)$ & $0.0385(6)$ \\
\hline & & & 24 & $0.197(1)$ & $0.0427(4)$ \\
\hline & & & 28 & $0.1913(5)$ & $0.0434(3)$ \\
\hline & & & 32 & $0.1914(5)$ & $0.0443(2)$ \\
\hline & & & $\infty$ & $0.1900(7) \quad 2.52$ & $0.0444(2) \quad 1.24$ \\
\hline \multirow[t]{5}{*}{8} & 2.68 & 0.0103 & 20 & $0.239(3)$ & $0.0339(5)$ \\
\hline & & & 24 & $0.209(1)$ & $0.0396(4)$ \\
\hline & & & 28 & $0.1999(9)$ & $0.0415(3)$ \\
\hline & & & 32 & $0.1983(5)$ & $0.0417(1)$ \\
\hline & & & $\infty$ & $0.1964(8) \quad 0.87$ & $0.0421(2) \quad 0.36$ \\
\hline \multirow[t]{5}{*}{9} & 2.49 & 0.0100 & 28 & $0.196(1)$ & $0.0294(2)$ \\
\hline & & & 32 & $0.181(1)$ & $0.0320(2)$ \\
\hline & & & 36 & $0.1771(9)$ & $0.0329(2)$ \\
\hline & & & 40 & $0.1756(5)$ & $0.0324(2)$ \\
\hline & & & $\infty$ & $0.1740(6) \quad 0.59$ & $0.0330(1) \quad 6.04$ \\
\hline \multirow[t]{6}{*}{10} & 2.30 & 0.0112 & 28 & $0.228(1)$ & $0.0238(2)$ \\
\hline & & & 32 & $0.194(2)$ & $0.0257(3)$ \\
\hline & & & 36 & $0.180(1)$ & $0.0273(2)$ \\
\hline & & & 40 & $0.174(1)$ & $0.0277(1)$ \\
\hline & & & 48 & $0.1704(5)$ & $0.02813(8)$ \\
\hline & & & $\infty$ & $0.1699(6) \quad 0.45$ & $\begin{array}{ll}0.02807(9) \quad 2.88 \\
\end{array}$ \\
\hline
\end{tabular}

Table 1. Volume dependence of $m_{\pi}$ and $f_{\pi}$ and fixed lattice spacing and fermion mass, together with the infinite volume extrapolated results using (3.1) and (3.2). The $\chi^{2} /$ dof of the extrapolations are also shown.

volume effects are thus enhancing each other in the ratio and too small volumes will lead to an overestimation of $m_{\varrho} / f_{\pi}$.

An upper bound on the size of finite volume effects sets a lower bound on $m_{\pi} L$ for each $N_{f}$. The results in [37] have shown that this lower bound is heavily $N_{f}$-dependent in the range $2 \leq N_{f} \leq 6$. In the present work these finite volume investigations are extended to $7 \leq N_{f} \leq 10$.

The main low energy quantities $m_{\pi}$ and $f_{\pi}$ are measured at fixed lattice spacing and mass $m$ for various lattice volumes, since these observables are expected to be the most sensitive to the finite volume. The $m_{\pi} L$ dependence of these quantities are given by

$$
\begin{aligned}
m_{\pi}(L) & =m_{\pi \infty}+C_{m} g\left(m_{\pi \infty} L\right) \\
f_{\pi}(L) & =f_{\pi \infty}-C_{f} g\left(m_{\pi \infty} L\right),
\end{aligned}
$$

with some $m_{\pi \infty}, f_{\pi \infty}, C_{m}$ and $C_{f}$ parameters. The details follow the procedure explained in [37], in particular we have

$$
g(x)=\frac{4}{x} \sum_{n \neq 0} \frac{K_{1}(n x)}{n}
$$

in terms of the Bessel function $K_{1}$. The sum is over integers $\left(n_{1}, n_{2}, n_{3}, n_{4}\right)$ with $n^{2}=n_{1}^{2}+n_{2}^{2}+n_{3}^{2}+4 n_{4}^{2} \neq 0$ where $\mu=4$ corresponds to the time direction. The func- 

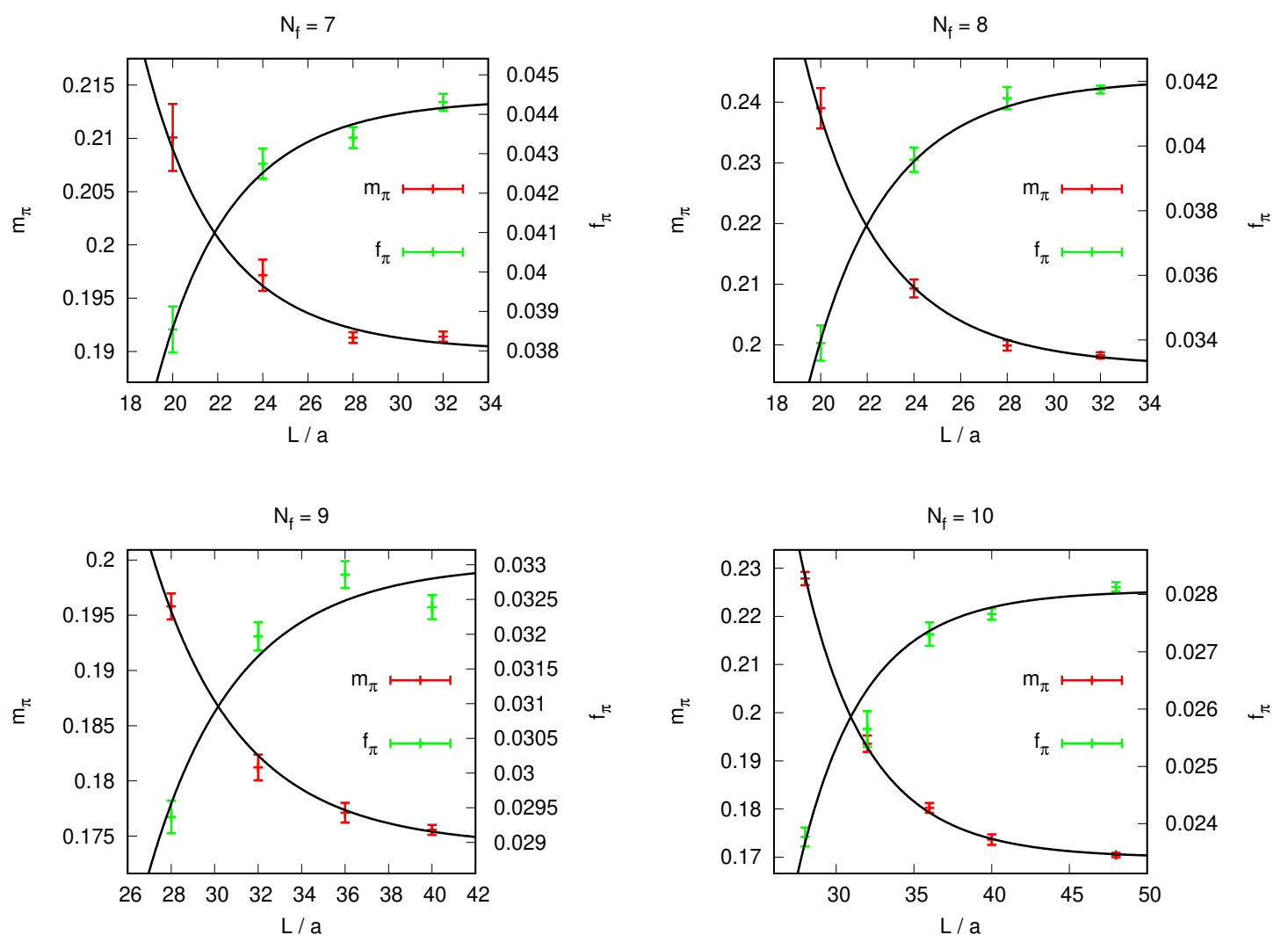

Figure 3. Finite volume effects for $m_{\pi}$ and $f_{\pi}$ for all flavors $7 \leq N_{f} \leq 10$. Extrapolations are via (3.2) and the data is tabulated in table 1.

tion $g(x)$ describing the finite volume effects represents the lightest particle, the pion, going around the finite volume in all 3 space and the time direction any number of times. The leading contribution for our geometry, where the lattice is largest in the time direction comes from the pion going around each spatial direction once, corresponding to $n=( \pm 1,0,0,0),(0, \pm 1,0,0),(0,0, \pm 1,0)$. If only these terms are kept we are led to the familiar finite volume effects given by a single exponential,

$$
g(x)=24 \sqrt{\frac{\pi}{2}} \frac{e^{-x}}{x^{3 / 2}}\left(1+O\left(\frac{1}{x}\right)\right)+O\left(e^{-\sqrt{2} x}\right) .
$$

We have repeated the finite volume fits with the above leading order single exponential expression as well and the results did not change within statistical uncertainties hence the data can not distinguish between the two sets of extrapolations. Note that the finite volume extrapolations (3.1) with either (3.2) or (3.3) do not depend on chiral perturbation theory at all, they hold for any massive QFT with $m_{\pi}$ taking the place of the lightest mass. In particular even if $N_{f}$ is inside the conformal window but a finite mass is introduced leading to finite masses for physical excitations, finite volume effects are still described by (3.1) and (3.2) or approximately (3.3). If on the other hand $N_{f}$ is outside the conformal window 2-loop results from chiral perturbation theory are available for the finite volume effects [47].

The results of our fits of the type (3.1) with (3.2) are shown in figure 3. 
The main conclusion from the finite volume study is that as $N_{f}$ is increasing the minimal $m_{\pi} L$ required for at most $1 \%$ finite volume effects needs to grow. On the full range $2 \leq N_{f} \leq 10$ including the results from [37] the bounds can be interpolated by the simple expression,

$$
m_{\pi} L>3.46+0.12 N_{f}+0.03 N_{f}^{2} .
$$

For instance at $N_{f}=10$ we have $m_{\pi} L>7.66$, about twice as large as the corresponding bound at $N_{f}=2$.

Apart from the exponential finite volume effects discussed above for $f_{\pi}$ and $m_{\pi}$, there might be further finite volume effects influencing $m_{\varrho}$ because of its possible decay to 2 pions. For our simulation points $\varrho$ is however stable.

The conclusion from this section is that our simulation results suffer from at most $1 \%$ finite volume effects for $N_{f}=7,8,9$ and at most $1.5 \%$ for $N_{f}=10$, resulting in at most $3 \%$ distortion in the ratio $m_{\varrho} / f_{\pi}$, well below our statistical uncertainties.

\section{Chiral-continuum extrapolation}

Apart from the observables $m_{\pi}, f_{\pi}$ and $m_{\varrho}$ the gradient flow scale $t_{0}$ was also measured to set the scale in the chiral-continuum extrapolations. The right hand side in the definition of $t_{0}[48]$,

$$
\left\langle t_{0}^{2} E\left(t_{0}\right)\right\rangle=c
$$

is in principle arbitrary, in QCD usually $c=0.3$ is used. It is possible to choose different $c=c\left(N_{f}\right)$ for different $N_{f}$ though. A combination of cut-off effects, statistical uncertainty and computational resources led to our following choices $c(7)=0.45, c(8)=0.45$, $c(9)=0.40, c(10)=0.32$.

Once $t_{0}$ is measured along with our low energy quantities of interest the chiralcontinuum extrapolation is performed via

$$
X \sqrt{t_{0}}=C_{0}+C_{1} m_{\pi}^{2} t_{0}+C_{2} \frac{a^{2}}{t_{0}}+C_{3} \frac{a^{2}}{t_{0}} m_{\pi}^{2} t_{0}
$$

where $X=f_{\pi}$ or $m_{\varrho}$. The continuum mass dependence is given by $C_{0}+C_{1} m_{\pi}^{2} t_{0}$ and the two terms $C_{2}$ and $C_{3}$ parametrize cut-off effects in both the chiral limit value $C_{0}$ and the slope $C_{1}$.

The measured data for $m_{\pi}, f_{\pi}, m_{\varrho}, t_{0}$ are shown in figure 3 . The lattice geometry was always $L^{3} \times 2 L$, the collected number of thermalized configurations $O(1000)$ and every $10^{\text {th }}$ was used for measurements. For each flavor number, simulations are performed at 3 lattice spacings with 4 masses at each. Hence the chiral-continuum extrapolations (4.2) correspond to $\operatorname{dof}=8$ in each case.

The chiral-continuum extrapolations are shown in figure 4 and the results are tabulated in table 2. The full $N_{f}$-dependence of $m_{\varrho} / f_{\pi}$ in the chiral-continuum limit for $2 \leq N_{f} \leq 10$ using also the results from [37] is shown in figure 5.

It was observed in [37] that there is no statistically significant $N_{f}$-dependence in the ratio for $2 \leq N_{f} \leq 6$, at least on the level of precision available there. A statistically good 

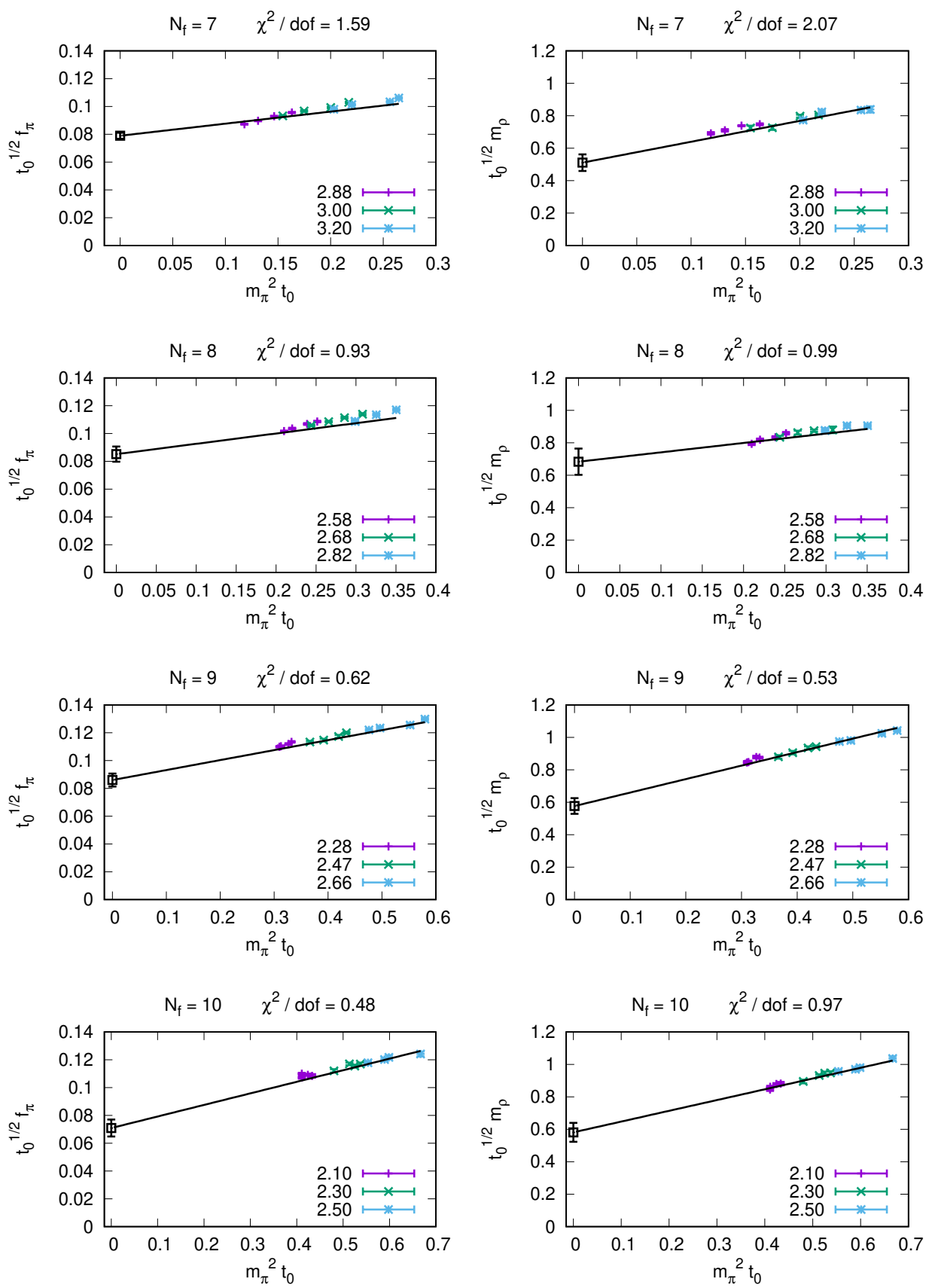

Figure 4. Chiral-continuum extrapolation of $f_{\pi}$ and $m_{\varrho}$ in $t_{0}$ units using the 4-parameter global fit (4.2). The $\chi^{2} /$ dof of the extrapolation is also shown. The solid black line corresponds to the resulting continuum mass dependence $C_{0}+C_{1} m_{\pi}^{2} t_{0}$, i.e. dropping $C_{2}$ and $C_{3}$ which are responsible for the cut-off effects. The deviations from the data at given bare coupling $\beta$ shown by different colors, and the straight line are indicative of said cut-off effects. The absolute scale on the axis can not be directly compared between different flavor numbers because the definition of $t_{0}$ was $N_{f}$-dependent, see (4.1). 


\begin{tabular}{|c|c|c|c|}
\hline$N_{f}$ & $f_{\pi} \sqrt{t_{0}}$ & $m_{\varrho} \sqrt{t_{0}}$ & $m_{\varrho} / f_{\pi}$ \\
\hline 7 & $0.079(2)$ & $0.51(5)$ & $6.5(7)$ \\
\hline 8 & $0.085(5)$ & $0.68(8)$ & $8.0(1.1)$ \\
\hline 9 & $0.086(5)$ & $0.58(5)$ & $6.7(7)$ \\
\hline 10 & $0.071(6)$ & $0.58(6)$ & $8.2(1.1)$ \\
\hline
\end{tabular}

Table 2. Continuum results for each $N_{f}$ in the chiral limit.

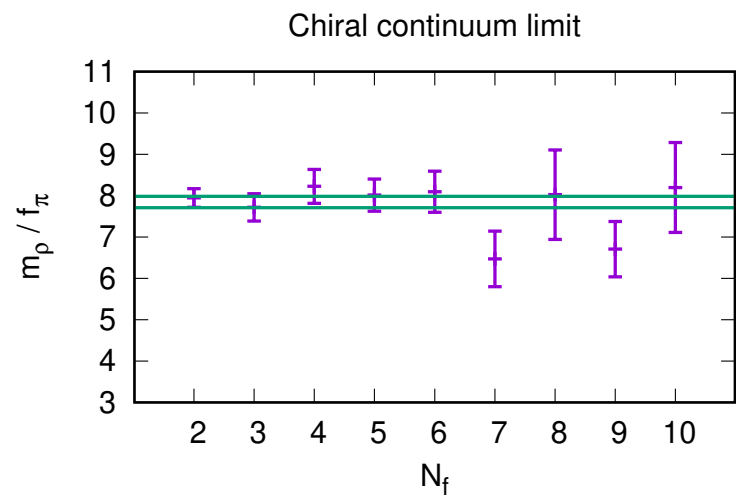

Figure 5. The $N_{f}$-dependence of $m_{\varrho} / f_{\pi}$ in the chiral-continuum limit. The results with $2 \leq N_{f} \leq 6$ are from [37] and $7 \leq N_{f} \leq 10$ corresponds to this work. The result of a constant fit as a function of $N_{f}$ is also shown.

constant fit gave $m_{\varrho} / f_{\pi}=7.95(15)$. We can now repeat the constant fit on the new range $7 \leq N_{f} \leq 10$ and the result is $m_{\varrho} / f_{\pi}=7.01(40)$ with $\chi^{2} /$ dof $=0.97$, which represents a slight $2 \sigma$-decrease. Nevertheless combining all results on the full range $2 \leq N_{f} \leq 10$ we obtain $m_{\varrho} / f_{\pi}=7.85(14)$ with $\chi^{2} / d o f=1.10$ which is our final result. ${ }^{1}$ Apparently, the free value $m_{\varrho} / f_{\pi}=1 / \sqrt{3}$ at $N_{f}=33 / 2$ is still about an order of magnitude away.

\section{Conclusion}

In this work we continued our study of the ratio $m_{\varrho} / f_{\pi}$ in the chiral-continuum limit. Constant fits as a function of $N_{f}$ on the two ranges $2 \leq N_{f} \leq 6$ and $7 \leq N_{f} \leq 10$ show a decrease on the $2 \sigma$-level but a constant fit on the full range $2 \leq N_{f} \leq 10$ is still a statistically acceptable result and leads to $m_{\varrho} / f_{\pi}=7.85(14)$.

The main conclusion is the reinforcement of the picture arising from [37], namely that $m_{\varrho} / f_{\pi}$ is a robust quantity once the gauge group is fixed and does not depend much, if at all, on the fermion content. Applied to composite Higgs models inspired by strong dynamics, this would mean that a potential measurement of a new so far unobserved vector resonance

\footnotetext{
${ }^{1}$ As a consistency check we have also fitted the $N_{f}$-dependence as $m_{\varrho} / f_{\pi}=A+N_{f} B$ which resulted in $A=8.17(29), B=-0.083(66)$ with $\chi^{2} /$ dof $=1.04$. The fit parameter $B$ is consistent with zero on the $1.3 \sigma$ level.
} 


\begin{tabular}{|c|c|c|c|c|c|c|c|c|c|}
\hline$N_{f}$ & $\beta$ & $m$ & $L / a$ & $a m_{\pi}$ & $a f_{\pi}$ & $a m_{\varrho}$ & $t_{0} / a^{2}$ & $m_{\pi} L$ & $f_{\pi} L$ \\
\hline \multirow[t]{12}{*}{7} & 2.88 & 0.0149 & 24 & $0.2583(5)$ & $0.0612(2)$ & $0.478(3)$ & $2.45(2)$ & $6.20(1)$ & $1.469(5)$ \\
\hline & & 0.0122 & 28 & $0.2327(4)$ & $0.0565(1)$ & $0.449(2)$ & $2.70(2)$ & $6.52(1)$ & $1.583(4)$ \\
\hline & & 0.0100 & 28 & $0.2092(4)$ & $0.0519(1)$ & $0.410(4)$ & $2.99(2)$ & $5.86(1)$ & $1.452(4)$ \\
\hline & & 0.0086 & 32 & $0.1932(4)$ & $0.04904(9)$ & $0.389(4)$ & $3.17(2)$ & $6.18(1)$ & $1.569(3)$ \\
\hline & 3.00 & 0.0147 & 28 & $0.2353(8)$ & $0.0520(2)$ & $0.406(3)$ & $3.92(2)$ & $6.59(2)$ & $1.455(4)$ \\
\hline & & 0.0125 & 28 & $0.2164(4)$ & $0.0480(2)$ & $0.386(4)$ & $4.28(4)$ & $6.06(1)$ & $1.345(4)$ \\
\hline & & 0.0100 & 32 & $0.1914(5)$ & $0.0443(2)$ & $0.333(6)$ & $4.77(3)$ & $6.12(2)$ & $1.418(7)$ \\
\hline & & 0.0084 & 36 & $0.1738(5)$ & $0.0412(1)$ & $0.320(6)$ & $5.12(3)$ & $6.26(2)$ & $1.484(5)$ \\
\hline & 3.20 & 0.0115 & 36 & $0.1741(6)$ & $0.0359(2)$ & $0.284(6)$ & $8.74(6)$ & $6.27(2)$ & $1.292(6)$ \\
\hline & & 0.0100 & 36 & $0.1630(8)$ & $0.0333(2)$ & $0.269(4)$ & $9.65(6)$ & $5.87(3)$ & $1.198(7)$ \\
\hline & & 0.0085 & 40 & $0.1479(3)$ & $0.0319(2)$ & $0.260(4)$ & $10.07(9)$ & $5.92(1)$ & $1.277(7)$ \\
\hline & & 0.0077 & 40 & $0.1403(5)$ & $0.0305(2)$ & $0.241(3)$ & $10.3(1)$ & $5.61(2)$ & $1.219(7)$ \\
\hline \multirow[t]{12}{*}{8} & 2.58 & 0.0149 & 24 & $0.2619(5)$ & $0.0567(2)$ & $0.448(4)$ & $3.67(3)$ & $6.28(1)$ & $1.361(5)$ \\
\hline & & 0.0124 & 28 & $0.2355(5)$ & $0.0515(2)$ & $0.402(2)$ & $4.30(5)$ & $6.59(1)$ & $1.442(4)$ \\
\hline & & 0.0099 & 28 & $0.2096(8)$ & $0.0463(1)$ & $0.366(3)$ & $5.01(5)$ & $5.87(2)$ & $1.296(4)$ \\
\hline & & 0.0087 & 32 & $0.1945(5)$ & $0.0432(1)$ & $0.337(3)$ & $5.55(3)$ & $6.23(2)$ & $1.383(4)$ \\
\hline & 2.68 & 0.0145 & 28 & $0.2400(7)$ & $0.0492(2)$ & $0.380(8)$ & $5.35(5)$ & $6.72(2)$ & $1.378(5)$ \\
\hline & & 0.0124 & 28 & $0.2207(6)$ & $0.0460(2)$ & $0.361(2)$ & $5.86(6)$ & $6.18(2)$ & $1.288(4)$ \\
\hline & & 0.0103 & 32 & $0.1983(5)$ & $0.0417(1)$ & $0.332(4)$ & $6.76(5)$ & $6.34(2)$ & $1.336(4)$ \\
\hline & & 0.0083 & 36 & $0.1750(5)$ & $0.0375(1)$ & $0.296(3)$ & $7.97(7)$ & $6.30(2)$ & $1.350(4)$ \\
\hline & 2.82 & 0.0120 & 36 & $0.1959(6)$ & $0.0387(2)$ & $0.300(3)$ & $9.13(8)$ & $7.05(2)$ & $1.394(6)$ \\
\hline & & 0.0100 & 36 & $0.1770(5)$ & $0.0352(1)$ & $0.281(3)$ & $10.4(1)$ & $6.37(2)$ & $1.269(5)$ \\
\hline & & 0.0080 & 36 & $0.1583(6)$ & $0.0314(3)$ & $0.253(4)$ & $11.9(2)$ & $5.70(2)$ & $1.132(9)$ \\
\hline & & 0.0075 & 40 & $0.1515(5)$ & $0.0302(1)$ & $0.243(2)$ & $13.0(1)$ & $6.06(2)$ & $1.207(5)$ \\
\hline \multirow[t]{12}{*}{9} & 2.28 & 0.0164 & 28 & $0.2672(5)$ & $0.0526(2)$ & $0.406(2)$ & $4.66(4)$ & $7.48(1)$ & $1.472(4)$ \\
\hline & & 0.0128 & 32 & $0.2311(5)$ & $0.0452(1)$ & $0.355(3)$ & $6.12(6)$ & $7.39(2)$ & $1.447(4)$ \\
\hline & & 0.0100 & 36 & $0.1994(3)$ & $0.0393(1)$ & $0.302(3)$ & $7.86(9)$ & $7.18(1)$ & $1.416(4)$ \\
\hline & & 0.0090 & 40 & $0.1875(2)$ & $0.03703(9)$ & $0.285(3)$ & $8.81(7)$ & $7.500(9)$ & $1.481(4)$ \\
\hline & 2.47 & 0.0140 & 32 & $0.2198(5)$ & $0.0400(1)$ & $0.315(2)$ & $8.98(8)$ & $7.03(2)$ & $1.281(4)$ \\
\hline & & 0.0110 & 36 & $0.1906(5)$ & $0.0345(2)$ & $0.275(2)$ & $11.5(2)$ & $6.86(2)$ & $1.243(6)$ \\
\hline & & 0.0090 & 40 & $0.1684(6)$ & $0.0309(2)$ & $0.244(2)$ & $13.8(2)$ & $6.74(2)$ & $1.234(7)$ \\
\hline & & 0.0070 & 48 & $0.1442(3)$ & $0.0270(1)$ & $0.210(2)$ & $17.6(2)$ & $6.92(2)$ & $1.296(5)$ \\
\hline & 2.66 & 0.0200 & 28 & $0.248(1)$ & $0.0423(2)$ & $0.340(1)$ & $9.4(1)$ & $6.95(3)$ & $1.185(7)$ \\
\hline & & 0.0150 & 32 & $0.2078(7)$ & $0.0351(2)$ & $0.287(2)$ & $12.8(2)$ & $6.65(2)$ & $1.124(7)$ \\
\hline & & 0.0120 & 40 & $0.1785(8)$ & $0.0313(2)$ & $0.249(2)$ & $15.6(3)$ & $7.14(3)$ & $1.250(7)$ \\
\hline & & 0.0098 & 48 & $0.1568(5)$ & $0.0277(2)$ & $0.222(2)$ & $19.3(2)$ & $7.53(2)$ & $1.331(7)$ \\
\hline \multirow[t]{12}{*}{10} & 2.10 & 0.0165 & 32 & $0.2423(7)$ & $0.0416(2)$ & $0.326(2)$ & $7.00(7)$ & $7.75(2)$ & $1.330(5)$ \\
\hline & & 0.0126 & 36 & $0.2037(7)$ & $0.0342(1)$ & $0.275(1)$ & $10.2(1)$ & $7.33(2)$ & $1.230(5)$ \\
\hline & & 0.0100 & 40 & $0.1801(9)$ & $0.0297(2)$ & $0.241(2)$ & $13.4(3)$ & $7.20(3)$ & $1.186(6)$ \\
\hline & & 0.0081 & 48 & $0.1535(6)$ & $0.02567(9)$ & $0.202(2)$ & $17.4(2)$ & $7.37(3)$ & $1.232(4)$ \\
\hline & 2.30 & 0.0185 & 32 & $0.2386(7)$ & $0.0390(1)$ & $0.310(1)$ & $9.0(1)$ & $7.63(2)$ & $1.247(5)$ \\
\hline & & 0.0142 & 36 & $0.2005(9)$ & $0.0320(1)$ & $0.259(2)$ & $13.4(2)$ & $7.22(3)$ & $1.152(5)$ \\
\hline & & 0.0112 & 40 & $0.174(1)$ & $0.0277(1)$ & $0.227(1)$ & $17.4(3)$ & $6.95(4)$ & $1.106(4)$ \\
\hline & & 0.0091 & 48 & $0.1502(9)$ & $0.0243(2)$ & $0.194(2)$ & $21.3(4)$ & $7.21(4)$ & $1.166(7)$ \\
\hline & 2.50 & 0.0233 & 28 & $0.2584(9)$ & $0.0393(3)$ & $0.328(2)$ & $10.0(2)$ & $7.23(3)$ & $1.100(8)$ \\
\hline & & 0.0178 & 36 & $0.2106(5)$ & $0.0332(2)$ & $0.267(2)$ & $13.5(3)$ & $7.58(2)$ & $1.194(8)$ \\
\hline & & 0.0141 & 40 & $0.1800(7)$ & $0.0282(1)$ & $0.227(2)$ & $18.2(3)$ & $7.20(3)$ & $1.128(6)$ \\
\hline & & 0.0114 & 48 & $0.1571(6)$ & $0.0249(1)$ & $0.202(1)$ & $22.4(2)$ & $7.54(3)$ & $1.195(7)$ \\
\hline
\end{tabular}

Table 3. Data used for the chiral-continuum extrapolations. The temporal extent of the lattices were always twice $L / a$. 
inherent in these types of models, would not select the flavor number. The measured vector mass would rather place constraints on the gauge group [49].

Our ratio has a well-defined meaning in the chiral limit both inside and outside the conformal window. If the free value $m_{\varrho} / f_{\pi}=1 / \sqrt{3}=0.577$ is to be reached at $N_{f}=16.5$, an order of magnitude drop ought to take place beyond $N_{f}=10$. If the trends of finite volume effects follow (3.4) in any sense, the $N_{f}>10$ simulations will be very challenging. It would be most interesting to work out the perturbative corrections to $1 / \sqrt{3}$ close to the upper end of the conformal window, i.e. not much below $N_{f}=16.5$ where perturbation theory is reliable. Hopefully the full range $2 \leq N_{f} \leq 16$ can then be covered by a combination of non-perturbative simulations and perturbative results. The onset of the conformal window would probably leave some sort of imprint on the flavor dependence of the ratio, a subject we leave for future work.

\section{Acknowledgments}

DN would like to thank very useful discussions with Stephan Durr and Sandor Katz. The simulations were carried out on the GPU clusters of Eotvos University Budapest and University of Wuppertal and at HLRS in Stuttgart, Germany. This work was in part supported by the Hungarian National Research, Development and Innovation Office (NKFIH) grant KKP126769.

Open Access. This article is distributed under the terms of the Creative Commons Attribution License (CC-BY 4.0), which permits any use, distribution and reproduction in any medium, provided the original author(s) and source are credited.

\section{References}

[1] W.A. Bardeen, C.T. Hill and M. Lindner, Minimal Dynamical Symmetry Breaking of the Standard Model, Phys. Rev. D 41 (1990) 1647 [InSPIRE].

[2] Z. Fodor, K. Holland, J. Kuti, D. Nogradi and C. Schroeder, Nearly conformal gauge theories in finite volume, Phys. Lett. B 681 (2009) 353 [arXiv:0907.4562] [INSPIRE].

[3] L. Del Debbio, B. Lucini, A. Patella, C. Pica and A. Rago, Mesonic spectroscopy of Minimal Walking Technicolor, Phys. Rev. D 82 (2010) 014509 [arXiv: 1004.3197] [INSPIRE].

[4] L. Del Debbio, B. Lucini, A. Patella, C. Pica and A. Rago, The infrared dynamics of Minimal Walking Technicolor, Phys. Rev. D 82 (2010) 014510 [arXiv:1004.3206] [InSPIRE].

[5] F. Bursa et al., Improved Lattice Spectroscopy of Minimal Walking Technicolor, Phys. Rev. D 84 (2011) 034506 [arXiv: 1104.4301] [INSPIRE].

[6] Z. Fodor, K. Holland, J. Kuti, D. Nogradi, C. Schroeder and C.H. Wong, Can the nearly conformal sextet gauge model hide the Higgs impostor?, Phys. Lett. B 718 (2012) 657 [arXiv: 1209.0391] [inSPIRE].

[7] T. Appelquist et al., Two-Color Gauge Theory with Novel Infrared Behavior, Phys. Rev. Lett. 112 (2014) 111601 [arXiv:1311.4889] [INSPIRE].

[8] LATKMI collaboration, Light composite scalar in twelve-flavor QCD on the lattice, Phys. Rev. Lett. 111 (2013) 162001 [arXiv:1305.6006] [INSPIRE]. 
[9] A. Hietanen, R. Lewis, C. Pica and F. Sannino, Fundamental Composite Higgs Dynamics on the Lattice: SU(2) with Two Flavors, JHEP 07 (2014) 116 [arXiv:1404.2794] [INSPIRE].

[10] LSD collaboration, Lattice simulations with eight flavors of domain wall fermions in SU(3) gauge theory, Phys. Rev. D 90 (2014) 114502 [arXiv: 1405.4752] [INSPIRE].

[11] LatKMI collaboration, Light composite scalar in eight-flavor QCD on the lattice, Phys. Rev. D 89 (2014) 111502 [arXiv:1403.5000] [INSPIRE].

[12] LATKMI collaboration, Lattice study of the scalar and baryon spectra in many-flavor QCD, Int. J. Mod. Phys. A 32 (2017) 1747010 [arXiv:1510.07373] [INSPIRE].

[13] T. DeGrand, Y. Liu, E.T. Neil, Y. Shamir and B. Svetitsky, Spectroscopy of SU(4) gauge theory with two flavors of sextet fermions, Phys. Rev. D 91 (2015) 114502 [arXiv: 1501.05665] [INSPIRE].

[14] L. Del Debbio, B. Lucini, A. Patella, C. Pica and A. Rago, Large volumes and spectroscopy of walking theories, Phys. Rev. D 93 (2016) 054505 [arXiv:1512.08242] [InSPIRE].

[15] LATKMI collaboration, Light flavor-singlet scalars and walking signals in $N_{f}=8 Q C D$ on the lattice, Phys. Rev. D 96 (2017) 014508 [arXiv:1610.07011] [INSPIRE].

[16] T. Appelquist et al., Strongly interacting dynamics and the search for new physics at the LHC, Phys. Rev. D 93 (2016) 114514 [arXiv:1601.04027] [InSPIRE].

[17] Z. Fodor, K. Holland, J. Kuti, S. Mondal, D. Nogradi and C.H. Wong, Electroweak interactions and dark baryons in the sextet BSM model with a composite Higgs particle, Phys. Rev. D 94 (2016) 014503 [arXiv: 1601.03302] [INSPIRE].

[18] T.A. DeGrand, M. Golterman, W.I. Jay, E.T. Neil, Y. Shamir and B. Svetitsky, Radiative contribution to the effective potential in composite Higgs models from lattice gauge theory, Phys. Rev. D 94 (2016) 054501 [arXiv:1606.02695] [INSPIRE].

[19] R. Arthur, V. Drach, M. Hansen, A. Hietanen, C. Pica and F. Sannino, SU(2) gauge theory with two fundamental flavors: A minimal template for model building, Phys. Rev. D 94 (2016) 094507 [arXiv: 1602.06559] [INSPIRE].

[20] Z. Fodor, K. Holland, J. Kuti, S. Mondal, D. Nogradi and C.H. Wong, Fate of the conformal fixed point with twelve massless fermions and SU(3) gauge group, Phys. Rev. D 94 (2016) 091501 [arXiv: 1607.06121] [INSPIRE].

[21] T. Appelquist, J. Ingoldby and M. Piai, Analysis of a Dilaton EFT for Lattice Data, JHEP 03 (2018) 039 [arXiv:1711.00067] [INSPIRE].

[22] T. Appelquist, J. Ingoldby and M. Piai, Dilaton EFT Framework For Lattice Data, JHEP 07 (2017) 035 [arXiv: 1702.04410] [inSPIRE].

[23] V. Ayyar et al., Spectroscopy of $\mathrm{SU}(4)$ composite Higgs theory with two distinct fermion representations, Phys. Rev. D 97 (2018) 074505 [arXiv:1710.00806] [INSPIRE].

[24] L. Del Debbio, C. Englert and R. Zwicky, A UV Complete Compositeness Scenario: LHC Constraints Meet The Lattice, JHEP 08 (2017) 142 [arXiv:1703.06064] [INSPIRE].

[25] Lattice Strong Dynamics collaboration, Nonperturbative investigations of SU(3) gauge theory with eight dynamical flavors, Phys. Rev. D 99 (2019) 014509 [arXiv:1807.08411] [INSPIRE].

[26] V. Ayyar et al., Partial compositeness and baryon matrix elements on the lattice, Phys. Rev. D 99 (2019) 094502 [arXiv: 1812.02727] [INSPIRE]. 
[27] V. Ayyar et al., Baryon spectrum of SU(4) composite Higgs theory with two distinct fermion representations, Phys. Rev. D 97 (2018) 114505 [arXiv: 1801.05809] [INSPIRE].

[28] T. Appelquist, J. Ingoldby and M. Piai, Dilaton potential and lattice data, Phys. Rev. D 101 (2020) 075025 [arXiv: 1908.00895] [INSPIRE].

[29] Z. Fodor, K. Holland, J. Kuti and C.H. Wong, Tantalizing dilaton tests from a near-conformal EFT, PoS LATTICE2018 (2019) 196 [arXiv:1901.06324] [INSPIRE].

[30] USQCD collaboration, Lattice Gauge Theory for Physics Beyond the Standard Model, Eur. Phys. J. A 55 (2019) 198 [arXiv: 1904.09964] [InSPIRE].

[31] V. Ayyar et al., Radiative Contribution to the Composite-Higgs Potential in a Two-Representation Lattice Model, Phys. Rev. D 99 (2019) 094504 [arXiv:1903.02535] [INSPIRE].

[32] Z. Fodor, K. Holland, J. Kuti and C.H. Wong, Dilaton EFT from p-regime to RMT in the $\epsilon$-regime, PoS LATTICE2019 (2020) 246 [arXiv: 2002.05163] [INSPIRE].

[33] Lattice Strong Dynamics collaboration, Near-conformal dynamics in a chirally broken system, Phys. Rev. D 103 (2021) 014504 [arXiv:2007.01810] [INSPIRE].

[34] A. Hasenfratz, C. Rebbi and O. Witzel, Gradient flow step-scaling function for SU(3) with ten fundamental flavors, Phys. Rev. D 101 (2020) 114508 [arXiv: 2004.00754] [INSPIRE].

[35] V. Drach, Composite electroweak sectors on the lattice, PoS LATTICE2019 (2020) 242 [arXiv:2005.01002] [INSPIRE].

[36] K. Cichy, J. Gonzalez Lopez, K. Jansen, A. Kujawa and A. Shindler, Twisted Mass, Overlap and Creutz Fermions: Cut-off Effects at Tree-level of Perturbation Theory, Nucl. Phys. B 800 (2008) 94 [arXiv: 0802.3637] [INSPIRE].

[37] D. Nogradi and L. Szikszai, The flavor dependence of $m_{\varrho} / f_{\pi}$, JHEP 05 (2019) 197 [arXiv: 1905.01909] [INSPIRE].

[38] C. Morningstar and M.J. Peardon, Analytic smearing of SU(3) link variables in lattice QCD, Phys. Rev. D 69 (2004) 054501 [hep-lat/0311018] [inSPIRE].

[39] S. Dürr et al., Lattice QCD at the physical point: Simulation and analysis details, JHEP 08 (2011) 148 [arXiv: 1011.2711] [INSPIRE].

[40] S. Duane, A.D. Kennedy, B.J. Pendleton and D. Roweth, Hybrid Monte Carlo, Phys. Lett. B 195 (1987) 216 [INSPIRE].

[41] M.A. Clark and A.D. Kennedy, Accelerating dynamical fermion computations using the rational hybrid Monte Carlo (RHMC) algorithm with multiple pseudofermion fields, Phys. Rev. Lett. 98 (2007) 051601 [hep-lat/0608015] [INSPIRE].

[42] W.-J. Lee and S.R. Sharpe, Partial flavor symmetry restoration for chiral staggered fermions, Phys. Rev. D 60 (1999) 114503 [hep-lat/9905023] [INSPIRE].

[43] C. Aubin and Q.-h. Wang, A Possible Aoki phase for staggered fermions, Phys. Rev. D 70 (2004) 114504 [hep-lat/0410020] [INSPIRE].

[44] A. Cheng, A. Hasenfratz and D. Schaich, Novel phase in SU(3) lattice gauge theory with 12 light fermions, Phys. Rev. D 85 (2012) 094509 [arXiv:1111.2317] [InSPIRE].

[45] C. Aubin, K. Colletti and G. Davila, Unphysical phases in staggered chiral perturbation theory, Phys. Rev. D 93 (2016) 085009 [arXiv:1512.01254] [INSPIRE]. 
[46] M.F.L. Golterman and J. Smit, Selfenergy and Flavor Interpretation of Staggered Fermions, Nucl. Phys. B 245 (1984) 61.

[47] J. Bijnens and T. Rössler, Finite Volume and Partially Quenched QCD-like Effective Field Theories, JHEP 11 (2015) 017 [arXiv:1509.04082].

[48] M. Lüscher, Properties and uses of the Wilson flow in lattice QCD, JHEP 08 (2010) 071 [Erratum ibid. 03 (2014) 092] [arXiv:1006.4518] [INSPIRE].

[49] D. Nogradi and L. Szikszai, The model dependence of $m_{\varrho} / f_{\pi}$, PoS LATTICE2019 (2019) 237 [arXiv: 1912.04114] [INSPIRE]. 